J. Clin. Chem. Clin. Biochem.

Vol. 18, 1980, pp. 227-23.2

\title{
Eine gaschromatographische Methode zur Bestimmung von Carbamazepin, Phenobarbital, Phenytoin und Primidon im gleichen Serumextrakt
}

\author{
Von W. R. Külpmann ${ }^{1}$ ) \\ Institut für Klinische Chemie (geschäftsf. Direktor Prof. Dr. Dr. J. Büttner) \\ Medizinische Hochschule Hannover
}

(Eingegangen am 16. Juli/20. Oktober 1979)

\begin{abstract}
Zusammenfassung: Es wird ein gaschromatographisches Verfahren zur Bestimmung der Antiepileptica Carbamazepin, Phenobarbital, Phenytoin und Primidon im gleichen Serumextrakt beschrieben. $1 \mathrm{ml}$ Serum wird nach Zusatz von gesättigter Ammoniumsulfatlösung mit Chloroform extrahiert. Die organische Phase wird abgetrennt, eingedampft und zur gaschromatographischen Analyse ohne Derivatbildung in $100 \mu \mathrm{l}$ Ethylacetat/Eisessig $(100 \mathrm{ml}+1 \mathrm{ml})$ aufgenommen. Als stationäre Phase für die Bestimmung von Phenobarbital und Phenytoin wird SP 2250 DA benutzt, für die Messung von Carbamazepin und Primidon Dexsil 300, jeweils unter isothermen Bedingungen. Der Variationskoeffizient für die Präzision von Tag zu Tag schwankt zwischen 4,3 und 7,5\%, die Wiederfindung beträgt 93,5 bis $111 \%$. Die Spezifität wird geprüft durch Vergleich mit den relativen Retentionszeiten von etwa 100 verschiedenen Arzneimitteln. Die Methode wurde mit entsprechenden enzymimmunologischen Verfahren verglichen.
\end{abstract}

\section{A gas-chromatographic method for the determination of carbamazepine, phenobarbital, phenytoin and primidone in the same extract of serum}

Summary: A gas-chromatographic method for the determination of the antiepileptic drugs carbamazepine, phenobarbital, phenytoin and primidone in the same extract of serum is presented. Saturated ammonium sulfate solution is added to $1 \mathrm{ml}$ serum, followed by extraction with chloroform. The organic phase is separated and evaporated. The residue is dissolved in $100 \mu \mathrm{l}$ ethylacetate/acetic acid $(100 \mathrm{ml}+1 \mathrm{ml})$ for gas-chromatography. The gas-chromatographic determination is carried out under isothermal conditions without derivatisation, using SP 2250 DA as stationary phase for the determination of phenobarbital and phenytoin, and Dexsil 300 for the determination of carbamazepine and primidone. The coefficient of variation for the precision from day to day ranges from 4.3 to $7.5 \%$, the recovery from 93.5 to $111 \%$. The specificity was proven by comparison with the relative retention times of about 100 drugs. The method is compared with the corresponding EMIT-tests.

\section{Einfiihrung}

Die Bedeutung der Konzentrations-Bestimmung von Antiepileptica im Serum für die Therapie der Anfallsleiden ist inzwischen weitgehend anerkannt. Die Analysen werden vorwiegend mit Hilfe der Gaschromatographie und in zunehmendem Maße mittels des homogenen Enzymimmunoassay (EMİT) ${ }^{2}$ ) durchgeführt. Wegen des vergleichsweise langsamen Meßverfahrens können nur

1) Auszugsweise vorgetragen auf der gemeinsamen Jahrestagung der Österreichischen Gesellschaft für Klinische Chemie und der Deutschen Gesellschaft fur Klinische Chemie, 29.-31. März 1979, Salzburg.

2) Hersteller: Syva; Palo Alto, Ca. U.S.A. Vertrieb in der Bundesrepublik Deutschland: Fa. E. Merck, Darmstadt. solche gaschromatographischen Verfahren an die Praktikabilität der EMIT-Tests heranreichen, welche die wichtigsten Anticonvulsiva gleichzeitig zu bestimmen erlauben (1-11), denn die EMIT-Tests gestatten nur die Bestimmung jeweils eines einzelnen Pharmakons, während häufig zur Behandlung verschiedene Antiepileptica gleichzeitig eingesetzt werden.

Die Praktikabilität der gaschromatographischen Verfahren läßt sich mittels Anwendung mechanisierter Probenaufgabesysteme und rechnerunterstützter Chromatogrammauswertung noch steigern. Der Einsatz dieser Hilfsmittel ist wesentlich erleichtert, wenn die gaschromatographische Analyse isotherm durchgefuhrt wird und die interessierenden Substanzen sich ohne Derivatbil- 
dung, insbesondere ohne Methylierung bestimmen lassen. Das im folgenden beschriebene gaschromatographische Verfahren gestattet die Bestimmung von Carbamazepin, Phenobarbital, Pheny toin und Primidon im gleichen Serumextrakt unter isothermen Bedingungen ohne Derivatbildung mittels Flammenionisationsdetektor. Es ist deshalb bei entsprechender Gaschromatographie-Ausrüstung ähnlich praktikabel wie die EMITTests.

\section{Material und Methodik}

\section{Material}

Carbamazepin (5H-dibenz[b,f]azepin-5-carboxamid) $M_{\mathrm{r}} 236,3$ (Geigy, Wehr)

Phenobarbital (5-Ethyl-5-phenylbarbitursäure) $M_{\mathrm{r}}$ 232,2 (Bayer, Leverkusen)

Phenytoin (5,5-Diphenylhydantoin) $M_{\mathrm{r}}$ 252,3 (Desitin, Hamburg) und Primidon (5-Ethyldihydro-5-phenyl-4,6- $(1 \mathrm{H}, 5 \mathrm{H})$-pyrimidin-dion) $M_{\mathrm{r}} 218,3$ (Desitin, Hamburg)

wurden von den angegebenen Firmen kostenlos zur Verfügung gestellt. Die Substanzen wurden in Ethylacetat/Eisessig $(100 \mathrm{ml}+1 \mathrm{ml})$ gelöst (Konzentration $1 \mathrm{~g} / \mathrm{l})$. Die Lösung ist bei $4{ }^{\circ} \mathrm{C}$ mindestens 3 Monate haltbar.

Natriumsulfat p.a., Ammoniumsulfat p.a. und Eisessig p.a. wurden wie von Merck, Darmstadt geliefert, verwendet. Ethylacetat und Chloroform wurden vor Gebrauch destilliert.

\section{Glasgeräte}

1. Schliffzentrifugenröhrchen mit spitzem Boden, Inhalt $25 \mathrm{ml}$.

2. Glassäulen $12 \mathrm{~cm}$ lang, $1 \mathrm{~cm}$ Innendurchmesser mit eingeschmolzener Glasfritte ohne Hahn.

Gaschromatographie: Gaschromatograph der Fa. Varian, Darmstadt, Modell 1440 und 2800, jeweils mit Flammenionisationsdetektor.

Trägergas: $\quad$ Nachgereinigter Stickstoff $35 \mathrm{ml} / \mathrm{min}$

Brenngase: $\quad$ Nachgereinigter Wasserstoff und synthetische Luft gefullt mit 3\% SP 2250 DA auf Supelcoport 100-120 mesh (Supelco, Bellefonte, USA) Säulenofentemperatur: $230^{\circ} \mathrm{C}$

2) 1,2 $\mathrm{m}$ lang, $2 \mathrm{~mm}$ Innendurchmesser gefüllt mit 3\% Dexsil 300 auf Supelcoport 100-120 mesh (Supelco, Bellefonte, USA)

Säulenofentemperatur: $210^{\circ} \mathrm{C}$
Glassäulen: $\quad$ 1) $1,8 \mathrm{~m}$ lang, $2 \mathrm{~mm}$ Innendurchmesser

\section{Methodik}

$1 \mathrm{ml}$ Serum wird mit $2 \mathrm{ml}$ kalt gesättigter Ammoniumsulfatlösung versetzt. Es wird eine Minute mit $5 \mathrm{ml}$ Chloroform ausgeschüttelt. Nach Zentrifugation $(5 \mathrm{~min}$ bei $3000 \mathrm{~g}$ ) wird die organische Phase abpipettiert und mittels Passage über die mit etwa $3 \mathrm{~g}$ Natriumsulfat gefüllte Glassäule getrocknet. Das Eluat wird aufgefangen. Die Extraktion wird zweimal wiederholt. Die vereinigten Eluate werden eingedampft. Der Rückstand wird in $100 \mu \mathrm{l}$ Ethylacetat/Eisessig $(100 \mathrm{ml}$ $+1 \mathrm{ml}$ ) gelöst und jeweils $2 \mu \mathrm{l}$ an den beiden Säulen injiziert. Die mit SP 2250 DA gefüllte Säule wird für die quantitative Bestimmung von Phenobarbital und Phenytoin benutzt, die Dexsil 300-haltige Säule für die Carbamazepin- und Primidonanalyse. Aus der Größe der Peakhöhen der Standards wird mittels der Gleichung der Regressionsgeraden die Konzentration des jeweiligen Antiepilepticums errechnet.

\section{Ergebnisse}

\section{Präzision}

Zur Bestimmung der Präzision in der Serie wurden jeweils $2 \mathrm{ml}$ eines żufällig ausgewählten Senums mit dem betreffenden Pharmakon aufgestockt, so daß Konzentrationen im therapeutischen Bereich erhalten wurden (12).

Jeweils $1 \mathrm{ml}$ der Probe wurde getrennt aufgearbeitet und analysiert. Die Variationskoeffizienten schwankten zwischen 3,5 und 4,4\% (Tab. 1).

Die Präzision von Tag zu Tag wurde bestimmt mit Hilfe eines selbst hergestellten Kontrollserums, das an 13 Tagen jeweils einmal analysiert wurde. Die relative Standardabweichung betrug zwischen 4,3 und $7,5 \%$ (Tab. 2).

Tab. 1. Wiederfindung und Präzision in der Serie.

\begin{tabular}{|c|c|c|c|c|c|}
\hline & $\begin{array}{l}\text { Anzahl } \\
\text { der } \\
\text { Analysen }\end{array}$ & $\begin{array}{l}\text { Sollwert } \\
(\mu \mathrm{mol} / \mathrm{l})\end{array}$ & $\begin{array}{l}\text { Mittel- } \\
\text { wert } \\
\bar{x} \\
(\mu \mathrm{mol} / \mathrm{l})\end{array}$ & $\begin{array}{l}\text { Ab- } \\
\text { weichung } \\
\text { vom } \\
\text { Sollwert }\end{array}$ & $\begin{array}{l}\text { Variations- } \\
\text { Koeffizient }\end{array}$ \\
\hline Substanz & $\mathbf{N}$ & & & $(\%)$ & (\%) \\
\hline $\begin{array}{l}\text { Carbama- } \\
\text { zepin }\end{array}$ & 8 & 21,2 & 22,9 & $+8,0$ & 3,5 \\
\hline $\begin{array}{l}\text { Pheno- } \\
\text { barbital }\end{array}$ & 8 & 86,1 & 85,9 & $-0,3$ & 4,4 \\
\hline Phenytoin & 8 & 79,3 & 74,2 & $-6,5$ & 4,2 \\
\hline Primidon & 9 & 22,9 & 25,4 & $+11,0$ & $4, \overline{3}$ \\
\hline
\end{tabular}

Tảb. 2. Präzision von Tag zu Tag.

Die Glassäulen wurden vor dem Füllen silikonisiert. Nur die Detektorseite wurde mit silikonisierter Glaswolle verschlossen. Insbesondere die Dexsil 300-Säule bedurfte vor der Verwendung einer sorgfältigen Konditionierung:

1. $30 \mathrm{~min}$ Raumtemperatur Trägergas $\mathrm{N}_{2} 35 \mathrm{ml} / \mathrm{min}$

2. $3^{\circ} \mathrm{C} / \mathrm{min}$ auf $150^{\circ} \mathrm{C}$ Trägergas $\mathrm{N}_{2} 35 \mathrm{ml} / \mathrm{min}$

3. $60 \mathrm{~min}$ bei $220^{\circ} \mathrm{C}$ Trägergas $\mathrm{N}_{2} 5 \mathrm{ml} / \mathrm{min}$

4. abkühlen auf $40^{\circ} \mathrm{C}$, dann ständig $\mathrm{N}_{2} 35 \mathrm{ml} / \mathrm{min}$

5. $3^{\circ} \mathrm{C} / \mathrm{min}$ auf $200^{\circ} \mathrm{C}$ Endtemperatur über Nacht

6. abkühlen auf $40^{\circ} \mathrm{C}$

7. $1^{\circ} \mathrm{C} / \mathrm{min}$ auf $310^{\circ} \mathrm{C}$, Endtemperatur über Nacht

8. abkühlen auf $40^{\circ} \mathrm{C}$

9. $1^{\circ} \mathrm{C} / \mathrm{min}$ auf $325^{\circ} \mathrm{C}$, Endtemperatur über Nacht

\begin{tabular}{lllll}
\hline & $\begin{array}{l}\text { Anzahl } \\
\text { der } \\
\text { Analysen }\end{array}$ & Sollwẹt & $\begin{array}{l}\text { Mittel- } \\
\text { wert } \\
(\mu \mathrm{mol} / 1)\end{array}$ & $\begin{array}{l}\text { Variations- } \\
\text { Koeffizient }\end{array}$ \\
Substanz & $\mathrm{N}$ & & & \\
\hline Carbamażepin & 13 & 21,2 & 22,7 & \\
Phenobarbital & 13 & 86,1 & 8,3 \\
Phenytoin & 13 & 79,3 & 78,1 & 5,1 \\
Primidon & 13 & 22,9 & 24,2 & 4,3 \\
\hline
\end{tabular}




\section{Rich tigkeit und Spezifität}

\section{Wiederfindung}

Die Wiederfindung wurde mit Hilfe von aufgestockten Sera ermittelt. Die prozentualen Abweichungen vom Sollwert lagen zwischen $-6,5$ und $+11 \%$ (Tab. 1).

\section{Patientenvergleich}

Es wurden Patientensera mit der beschriebenen Methode und mit EMIT-Tests bestimmt. Es ergab sich eine befriedigende Ubereinstimmung der Werte $(13,14)$.

Jeweils 1 Tablette oder Dragée bz̈w. $5 \mathrm{ml}$ der folgenden Medikamente wurde in $10 \mathrm{ml}$ Ethylacetat/Eisessig $(100 \mathrm{ml}+1 \mathrm{ml})$ gelöst und die relative Retentionszeit der extrahierten Substanzen in den verschiedenen gaschromatographischen Systemen ermittelt. Auf diese Weise wird die Löslichkeit von Pharmakon und Tablettensubstanz in dem für die Aufnahme des Extraktes verwendeten Lösungsmittel in einem Schritt mitberücksichtigt. Andererseits mußte deshalb auf eine Angabe der geprüften Konzentration der Reinsubstanz verzichtet werden:

Acidum acetylosalicylicum

Acidum ascorbicum

Acidum niflumicum

Adipiodon (Methylglucaminsalz $(5 \mathrm{ml}))$

Allopurinol

Amidotrizoesäure (Methylglucaminsalz $(5 \mathrm{ml}))$

Aminophenazon

Amitryptilin

Ampicillin

Antazolin

Azapropazon

Benzbromaron

Bisacodyl

Carbocromen

Carbromal

Cetobemidon

Chlordiazepoxid

Chloroquin

Chlorpromazin

Chlorprothixen

Clofibrat

Clomethiazol

Codein

Cyclophosphamid

Dextran $(5 \mathrm{ml})$

Dextromoramid

2,2-Diethylallylacetamid

Diazepam

Digoxin

Diphenhydramin

Doxepin

Ethinamat

Ethosuximid

Fenetyllin

Fluphenazin

Furosemid

Gentämy cin

Glafenin

Glibenclamid

Glutethimid

Hạlopereridôl

Hyoscin-N-butylbromid

Imipramin

Indometazin

Levorphanol

Levorphanoltartrat

Meclozin

Mephenytoin
Mesuximid
Methadon
Methamphetamin
Methaqualon
Methotrexat
Methyldopa
Methalpentynol
Methylstiryldibromhydantoin
Methyprylon
Miroton@
Modenol@
Morphin
Neoplasmagel (5 ml)
Nicotinamid
Nitrazepam
Nitrofurantoin
Noramidopyrini methano-
sulfonas natrium
Norfenefrin
Normethadon
Oxazepam
Oxyphenbutazon
Paramethadion
Pethidin
Phenformin
Pheniramin-p-amino-sälicylat
Phenprocoumon
Phenylbutazon
Prażepam
Prednisolon
Probenecid
Promethazin
Prophylhexedrin geb. an
Phenobarbital
Prothipendyl
Pyrithyldion
Reserpin
Spironolacton
Sulfadiazin
Sulfametoxydiazin
Sultiam
Tetracyclin
Thioridazin
Tilidin
Tolbutamid
Triflupromazin
Trimethadion
Valproinsäure

Wenn man die Retentionszeiten vergleicht, so könnte die Carbamazepinbestimmung gestört werden durch Prothipendyl, die von Phenobarbital durch Acetylsalicylsäure, Cyclophosphamid, Oxyphenbutazon und Normethadon, von Phenytoin durch Ascorbinsäure und Benzbromaron und von Primidon durch Doxepin, Imipramin und Triflupromazin. Auf Grund der geringen Serumkonzentrationen bei therapeutischer Dosierung brauchen von diesen Arzneistoffen nicht berücksichtigt zu werden: Doxepin, Imipramin, Normethadon, Prothipendyl und Triflupromazin. Die verbleibenden Pharmaka mit Ausnahme des Cyclophosphamid werden bei der Aufarbeitung abgetrennt. Mit der langwierigen Untersuchung der Störung durch Metabolite der Pharmaka wurde begonnen. Die Anticonvulsiva und ihre Metabolite, wie Phenylethylmalonsäurediamid und Carbamazepin-10,11epoxid werden vollständig voneinander getrennt. Bei Aufarbeitung von pharmakafreien Sera werden keine störenden Peaks beobachtet. Die Anticonvulsiva, auch Carbamazepin, ergeben an der jeweiligen stationären Phase einen einzigen Gipfel ohne Hinweise auf Zersetzung. Typische Gaschromatogramme sind auf den Abbildungen 1-3 dargestellt.

\section{Nachweisgrenze}

Die Bestimmung der Nachweisgrenze für gaschromatographische Verfahren ist nicht einheitlich geregelt. Die häufig gemachte Angabe des Signal-Rauschen-Verhältnisses mit Hilfe von Reinsubstanzen ergibt zu günstige Werte, da die Verunreinigungen, die aus dem biologischen Ausgangsmaterial stammen, und die Verluste bei der Aufarbeitung nicht berücksichtigt werden. Die Messung von Leerproben ist nicht möglich, zumal die Retentionszeit im niedrigsten Konzentrationsbereich nicht genau festzulegen ist. Wir haben deshalb - einer Empfehlung der IFCC folgend (15) - angenommen, daß die Standardabweichung (s) bei niedriger Substanzkonzentration etwa der von Leerproben entspricht und den entsprechenden $3 \mathrm{~s}$-Bereich errechnet. Über die Größe des mittleren Leerwerts können wir keine Angaben machen; als Anhalt sind in der Tabelle 3 der Sollwert und der gefundene Wert gegenübergestellt.

\section{Prak tikabilität}

Die Analyse einer Serumprobe in Bezug auf die 4 Substanzen erfordert etwa 2 Stunden. 20 Serumproben können in 10 Stunden analysiert werden. Davon entfallen etwa 8 Stunden auf die Durchführung der Gaschromatographie. Bei Verwendung von automatischem Probengeber und on-line Datenverarbeitung vermindert sich diese Zeit entsprechend. Isotherme Arbeitsweise und der Verzicht auf die Derivatbildung erleichtern diesen Rationalisierungsschritt. Nach der erforderlichen einmaligen Konditionierung der stationären Phase Dexsil 300, die an den meisten Geräten weitgehend 


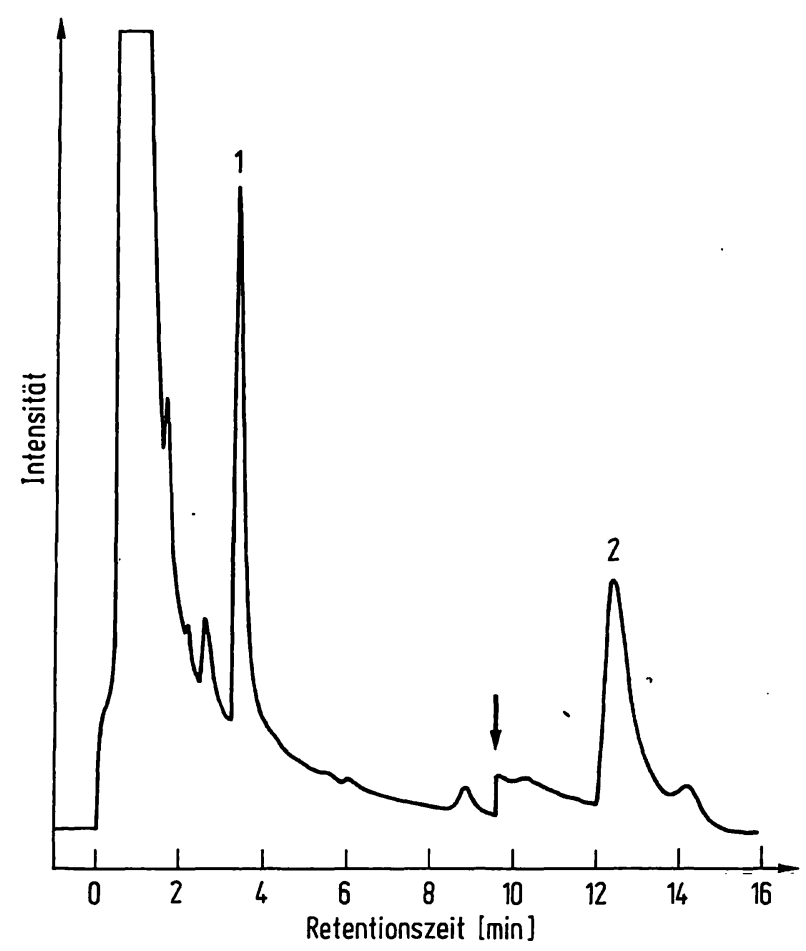

Abb. 1. Gaschromatogramm eines Serums von einem Patienten an SP 2250 DA. Die Konzentration an Phenobarbital (1) betrug $56 \mu \mathrm{mol} / 1$ (therapeutischer Bereich: $21,5-172,3$ $\mu \mathrm{mol} / 1$ (12)), an Phenytoin (2) $22 \mu \mathrm{mol} / 1$ (therapeutischer Bereich 19,8-99,1 $\mu \mathrm{mol} / 1$ (12)).

$\downarrow$ : Einstellung einer größeren Empfindlichkeit. unter der Behandlung mit Phenobarbital und Phenytoin

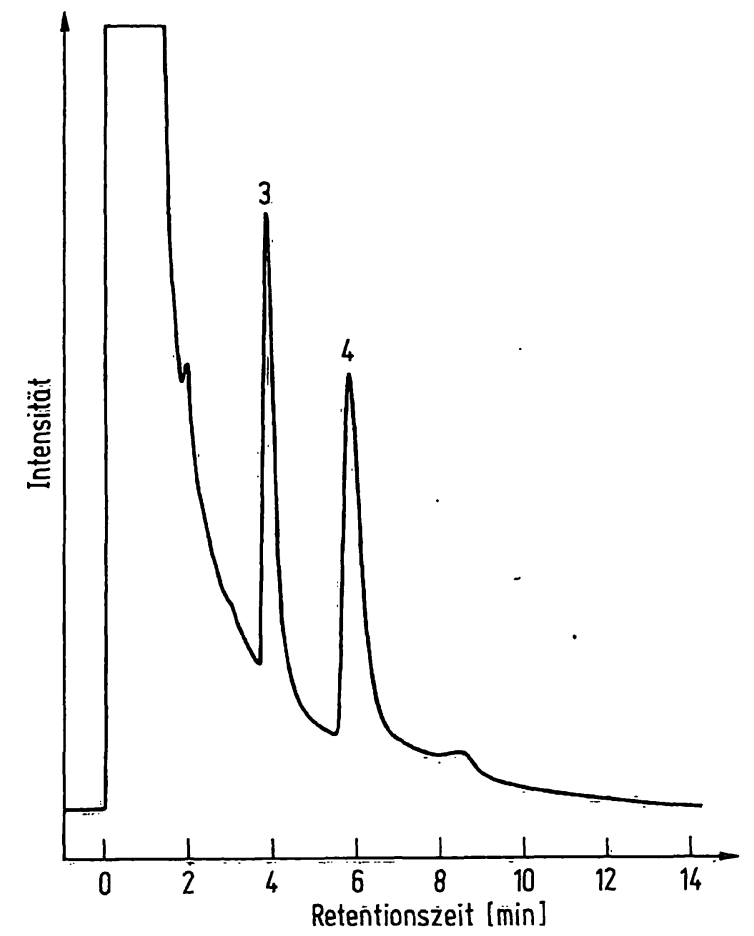

Abb. 3. Gaschromatogramm eines Serum von einem Patienten unter der Behandlung mit Primidon und Carbamazepin an Dexsil 300. Die Konzentration an Primidon (3) betrug $27,5 \mu \mathrm{mol} / 1$ (therapeutischer Bereich 22,9-91,6 $\mu \mathrm{mol} / 1$ (12)) und an Carbamażepin (4) $22,0 \mu \mathrm{mol} / 1$ (therapeutischer Bereich 12,7-50,8 $\mu \mathrm{mol} / 1$ (12)).

Phenobarbital ist im „Lösungsmittel-Tailing“ enthalten.

Tab. 3. 3 s-Bereich bei niedriger Substanzkonzentration (Nachweisgrenze).

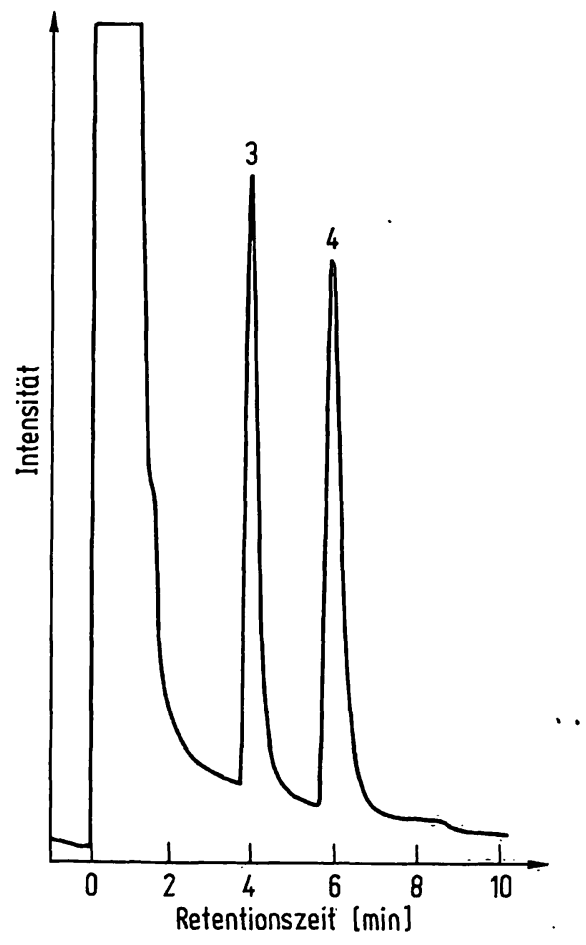

Abb. 2. Gaschromatogramm von $0,69 \mathrm{nmol}(150 \mathrm{ng}$ ) Primidon (3) und von $0,63 \mathrm{nmol}$ (150 ng) Carbamazepin (4) an Dexsil 300.

\begin{tabular}{|c|c|c|c|c|c|}
\hline $\begin{array}{l}\text { Sub- } \\
\text { stanz }\end{array}$ & $\begin{array}{l}\text { Anzahl } \\
\text { der } \\
\text { Analysen }\end{array}$ & Sollwert & $\begin{array}{l}\text { Mittel- } \\
\text { wert } \\
\overline{\mathrm{x}}\end{array}$ & $\begin{array}{l}\text { Nach- } \\
\text { weis- } \\
\text { grenze } \\
\text { (3s- } \\
\text { Bereich) } \\
(\mu \mathrm{mol} / 1)\end{array}$ & $\begin{array}{l}(\mu \mathrm{mol} / 1) \\
\text { N. Schmidt (12) }\end{array}$ \\
\hline $\begin{array}{c}\text { Carbama- } \\
\text { zepin }\end{array}$ & 8 & 8,5 & 8,1 & 2,1 & $12,7-50,8$ \\
\hline $\begin{array}{l}\text { Pheno- } \\
\text { barbital }\end{array}$ & 8 & 21,5 & 19,4 & 10,3 & $21,5-172,3$ \\
\hline $\begin{array}{l}\text { Pheny- } \\
\text { toin }\end{array}$ & 10 & 19,8 & 17,2 & 7,2 & $19,8-99,1$ \\
\hline Primidon & 10 & 9,2 & 9,6 & 1,8 & $22,9-91,6$ \\
\hline
\end{tabular}

selbsttätig ablaufen kann, wurde sie Monate lang ohne Veränderung ihrer Trenneigenschaften benutżt. Bei Verwendung eines mechanisierten gaschromatographischen Arbeitsplatzes entsprechen die Personalkosten grob denen, die zur Durchführung der Analyse mit Hilfe der EMIT-Tests anfallen. Dabēi ist berücksichtigt, daß die gaschromatographische Methode vièr verschiedene Antiepileptika im gleichen Extrakt zu messen gestattet, während der EMIT-Test nur jeweils éin Pharmakon zu bestimmen erlaubt. Die Reagentien für die gáśchromatô- 
graphische Analyse sind vergleichsweise billig (etwa $0,35 \mathrm{DM}$ je Analyse) und jederzeit verfügbar. Die Investitionskosten für einen mechanisierten gaschromatographischen Meßplatz entsprechen in der gleichen Ausbaustufe etwa denen für einen mechanisierten EMITMeßplatz.

\section{Diskussion}

Das beschriebene Verfahren ergibt nach einfacher Probenvorbereitung von störenden Verunreinigungen freie Extrakte. Die in dieser Arbeit verwendeten stationären Phasen waren für die verschiedenen Antiepileptica nicht gleich gut geeignet. Pheny toin ergab unbefriedigende Gaschromatogramme an Dexsil 300, Carbamazepin und Primidon an SP 2250 DA. Phenobarbital kann an beiden Systemen analysiert werden. Die Verwendung von Dexsil 300 erlaubt die zerstörungsfreie Gaschromatographie von unverändertem Carbamazepin. Die gaschromatographische Analyse wird durchgeführt isotherm ohne Derivatbildung unter Verwendung eines Flammenionisationsdetektors und bietet damit, im Gegensatz zu anderen gaschromatographischen Verfahren, optimale Voraussetzungen für eine Mechanisierung. Unter Ausschluß der Verfahren mit Derivatbildung ist dagegen bei der Methode nach Beam (2) eine Temperaturprogrammierung vorgesehen. Carbamazepin wird nicht erfaßt. Bei dem Verfahren nach Bredesen \& Johannessen (3) beträgt die Wiederfindung von Primidon lediglich knapp $50 \%$, die Auftrennung von Primidon, Carbamazepin und Phenytoin ist unbefriedigend. Die Bestimmung nach Toseland (4) und die Weiterentwicklung dieser Methode durch Heipertz (9) ist an die Verwendung eines $\mathrm{N}$-empfindlichen Detektors mit Temperaturprogrammierung gebunden; etwa 20\% des Carbamazepin wird während der Gäschromatographie zerstört. Von Godolphin et al. (16) und Hewitt et al. (17) wird eine neue stationäre Phase - SP 2510 DA - verwendet, die für die gaschromatographische Bestimmung der Anticonvulsiva besser geeignet ist als die von Heipertz (9) benutzte SP 1000. Nachteilig ist, daß an SP 2510 DA Primidon und Cholesterin gleiche Retentionszeiten besitzen. Godolphin et al. (16) benutzen eine mit SP 2250 DA gefüllte Vorsäule zur Cholesterinabtrennung, eine Phase, die für die Bestimmung von Carbamazepin und Primidon nicht geeignet ist. Die Wiederfindung für diese Antiepileptica beträgt $80 \mathrm{bzw}$. 70\% (16). Hewitt et al. (17) trennen Cholesterin bei der Probenaufarbeitung ab. Bei diesem Verfahren beträgt die Wiederfindung von Primidon 69\%, so daß mehrere aufgestockte Leerșera in jeder Serie zur Korrektur mitgeführt werden müssen. Bei Verwendung eines Flammenionisationsdetektors bietet die Phase SP 2510 DA somit wegen der Schwierigkeiten bei der Primidon- bestimmung lediglich den Vorteil gegenüber der Phase SP 2250 DA, daß Carbamazepin zusätzlich bestimmt werden kann. Für toxikologische Analysen nach 1.c. (15) ist SP 2510 DA dagegen nicht geeignet. Steht ein Stickstoff-empfindlicher Detektor zur Verfügung, wird die Primidonbestimmung an SP 2510 DA durch Cholesterin nicht gestört. In diesem Fall ist die Verwendung von SP 2510 DA in Verbindung mit dem von uns angegebenen Aufarbeitungsverfahren wegen der konstant hohen Wiederfindung zur Bestimmung der vier Antiepileptica isotherm ohne Derivatbildung zu empfehlen.

Wegen der von Curry et al. (18) geäußerten Bedenken wurden die vorliegenden Ergebnisse ohne Bezug auf einen inneren Standard ermittelt. Auf Grund später durchgeführter Analysen ist zu erkennen, daß bei Zusatz von 5-(4'-Methylphenyl)-5-phenylhydantoin als innerem Standard die Präzision der Pheny toinbestimmung noch besser wird. Der Standard eignet sich - wie Phenytoin - nicht für die gaschromatographische Bestimmung an Dexsil 300.

Die Variationskoeffizienten als Maß für die Präzision von Tag zu Tag, berechnet unter Verwendung von Einzelwerten, sind kleiner als bei entsprechenden EMIT-Analysen $(13,14)$.

Beim Patienten-Vergleich mit dem EMIT-Test liegen die gaschromatographisch ermittelten Analysenergebnisse für Carbamazepin im Mittel um 0,8 $\mu \mathrm{mol} / 1$ niedriger, wahrscheinlich bedingt durch die Abtrennung von Carbamazepinepoxid, das bei der EMIT-Analyse miterfaßt wird.

Die Nachweisgrenze der gaschromatographischen Methode ist niedriger als die der EMIT-Tests.

Störungen z. B. durch Medikamente werden mit der gaschromatographischen Methode eher als bei enzymimmunologischen Verfahren erkannt.

In bezug auf die Reagentienkosten ist die gaschromatographische Bestimmung billiger, die Materialien sind haltbar und jederzeit verfügbar. Als Vorteil der EMITMethode ist anzusehen

1. daß gewöhnlich keine zusätzlichen Investitionskosten anfallen für die Einrichtung eines Meßplatzes, der zudem für eine Vielzahl anderer Analysen eingesetzt werden kann, da die Meßzeit kurz ist,

2. daß technische Mitarbeiter gewöhnlich eher Kenntnisse in der Photometrie als in der Gaschromatographie besitzen,

3. daß nur geringe Probenvolumina benötigt werden.

\section{Danksagung}

Herrn $K$. Petry und Herrn $W$. Werner danke ich für die zuverlässige Mitarbeit bei der Durchführung der Analysen. 


\section{Literatur}

1. Roger, J. C., Rodgers, G. Jr. \& Soo, A. (1973), Clin. Chem. 19, 590-592.

2. Beam, R. E. (1974), Am. J. Med. Technol. 40, 211-218.

3. Bredesen, J. E. \& Johannessen, S. I. (1974), Epilepsia 15, 611-617.

4. Toseland, P. A., Albani, M. \& Gauchel, F. D. (1975), Clin. Chem. 21, 98-103.

5. Least, C. J., Johnson, G. F. \& Solomon, H. M. (1975), Clin. Chem. 21, 1658-1622.

6. Davis, H. L., Falk, K. J. \& Bailey, D. G. (1975), J. Chromatogr. 107, 61-66.

7. Nishina, T., Okoshi, K. \& Kitamura, M. (1976), Clin. Chim. Acta 73, 463-468.

8. Abraham, C. V. \& Joslin, H. D. (1976), Clin. Chem. 22, 769-771.

9. Heipertz, R., Pilz, H. \& Eickhoff, K. (1977), Clin. Chim. Acta 77, 307-316.

10. Hill, R. E. \& Latham, A. N. (1977), J. Chromatogr. 131, $341-346$.
11. Malkus, H., Jatlow, P. I. \& Castro, A. (1978), Clin. Chim. Acta 82, 113-117.

12. Schmidt, D. (1977), Therapiewoche 27, 501-510.

13. Oellerich, M., Külpmann, W. R., Haeckel, R. \& Heyer, R. (1977), diese Z. 15, 353-358.

14. Külpmann, W. R. \& Oellerich, M. (1979), in Vorbereitung.

15. Büttner, J., Borth, R., Boutwell, J. H., Broughton, P. M. G. \& Bowyer, R. C. (1976), Clin. Chim. Acta 69, F1-F17; J. Clin. Chem. Clin. Biochem. 14 (1976), 265-275; Clin. Chem. 22 (1976), 1922-1932.

16. Godolphin, W. \& Thoma, J. (1978), Clin. Chem. 24, 483-485.

17. Hewitt, T. E., Sievers, D. L. \& Kessler, G. (1978), Clin. Chem. 24, 1854-1856.

18. Curry, S. H. \& Whelpton, R. (1978) in Blood Drugs and Other Analytical Challenges (Reid, E. ed.) S. 29-41, Verlag John Wiley \& Sons, New York.

Priv.-Doz. Dr. W. R. Külpmann Institut f. Klinische Chemie Karl-Wiechert-Allee 9 D-3000 Hannover 61 